[0212-7199 (2005) 22: 2; pp 79-81] ANALES DE MEDICINA INTERNA Copyright (C) 2005 ARAN EDICIONES, S.L

AN. MED. INTERNA (Madrid) Vol. 22, N. ${ }^{\circ} 2$, pp. 79-81, 2005

\title{
Dolor y amiotrofia en miembros superiores en una paciente cirrótica afecta de mal de Pott
}

\author{
E. GONZÁLEZ REIMERS, J, RUIZ LACAMBRA, J. LÓPEZ GARCÍA ${ }^{1}$, \\ M. RODRÍGUEZ GASPAR, J. L. MUÑIZ MONTES ${ }^{1}$, J. ABREU FALCÓN ${ }^{2}$, \\ F. SANTOLARIA FERNÁNDEZ
}

Servicios de Medicina Interna, 'Radiología y ${ }^{2}$ Fisioterapia. Hospital Universitario. Tenerife

PAIN AND AMYOTROPHY IN UPPER MEMBERS IN A CIRRHOTIC PATIENT WITH POTT'S DISEASE

\section{RESUMEN}

El síndrome de Parsonage-Turner o neuralgia amiotrófica del hombro es una entidad de etiología y patogenia desconocidas. Cursa con dolor intenso localizado en hombro y región proximal del miembro superior, especialmente nocturno, seguido de amiotrofia y debilidad. Se ha descrito en relación con infecciones víricas o bacterianas, procesos inflamatorios o intervenciones quirúrgicas, y suele tener una evolución favorable. Presentamos el caso de una paciente de 56 años de edad, afecta de cirrosis hepática de origen alcohólico, diagnosticada tres meses antes de espóndilodiscitis tuberculosa que afectaba a D9 y D10, que presenta de forma súbita dolor y amiotrofia en hombro y brazo derecho, seguido a las pocas semanas de afectación del lado izquierdo. Un estudio electrofisiológico confirmó el diagnóstico, y se realizó resonancia nuclear magnética, procedimiento diagnóstico útil en estas situaciones, especialmente cuando se planteaba el diagnóstico diferencial con tendinitis por quinolonas.

PALABRAS CLAVE: Neuralgia amiotrófica del hombro. Plexo braquial. Síndrome Parsonage-Turner.

\begin{abstract}
We report the case of a 56-year old cirrhotic woman who presented during the course of a tuberculous spondylodisctis affecting T9-T10, a clinical picture consistent with neuralgic amyotrophy affecting the right shoulder first, and later also the left one (Parsonage-Turner syndrome). This is an uncommonly diagnosed entity of unknown etiology and pathogenesis. Magnetic resonance images (MRI) include high signal intensity in supra and infraspinatus muscles and other muscles of the shoulder girdle, compatible with muscle oedema associated with denervation. These features, combined with the ability of MRI to exclude local problems as tendinitis stresses the importance of this technique in the diagnostic evaluation of patients with neuralgic amyotrophy.
\end{abstract}

KEY WORDS: Parsonage-Turner syndrome. Neuralgic amyotrophy. Brachial plexus.

González Reimers E, Ruiz Lacambra J, López García J, Rodríguez Gaspar M, Muñiz Montes JL, Abreu Falcón J, Santolaria Fernández F. Dolor y amiotrofia en miembros superiores en una paciente cirrótica afecta de mal de Pott. An Med Interna (Madrid) 2005; 22: 79-81.

\section{INTRODUCCIÓN}

El síndrome de Parsonage-Turner o neuralgia amiotrófica del hombro es una entidad de etiología y patogenia desconocidas $(1,2)$. Cursa con dolor intenso localizado en hombro y región proximal del miembro superior, especialmente nocturno, seguido de amiotrofia y pérdida de fuerza, en particular para la elevación y abducción del hombro. En un $25 \%$ de casos es bilateral. Se han descrito casos familiares, autosómicos dominantes, generalmente asociados a otras alteraciones congénitas como dismorfia facial con hipotelorismo y paladar hendido, sindactilia y estatura corta, o a la denominada polineuropatía tomaculosa, y casos esporádicos, que, aunque pueden ocurrir de forma aislada, sin aparente relación con procesos subyacentes, en más del $50 \%$ de ocasiones coexiste con procesos sistémicos graves como arteritis temporal, vacunaciones o infecciones víricas o bacterianas (1,3-5). A veces se afecta la inervación diafragmática, de forma uni o bilateral, y cursa con insuficiencia respiratoria aguda $(6,7)$. Suele ser reversible, aunque puede tardar años en recuperase, a veces no totalmente $(8,9)$. Puede ser bilateral, aunque rara vez simultáneo $(10)$. El diagnóstico es clínico y electromiográfico. Recientemente se ha visto que la resonancia magnética proporciona una imagen altamente sugestiva (11-13), de gran utilidad cuando ante la sintomatología inicial dolorosa se plantean otros diagnósticos, como en el caso que presentamos a continuación. 


\section{CASO APORTADO}

Paciente de 56 años de edad, afecta de cirrosis hepática de origen alcohólico, diagnosticada tres meses antes de espóndilodiscitis que afectaba a D9 y D10 y al disco intervertebral correspondiente. Por este motivo la paciente fue tratada con isoniacida 300 $\mathrm{mg} /$ día, rifampicina, $600 \mathrm{mg} /$ día, pirazinamida, $1.500 \mathrm{mg} /$ día y ciprofloxacino, $500 \mathrm{mg} / 12$ horas. Además, ante el riesgo de fractura completa del cuerpo vertebral y de compresión medular se indicó reposo en cama y se colocó un corsé. La evolución clínica y radiológica fue favorable a lo largo de estos meses, asistiéndose a una progresiva esclerosis de la lesión vertebral y tendencia a la fusión de ambas vértebras, con marcada disminución de la inflamación de partes blandas. Consulta ahora por presentar de forma subaguda -en el curso de uno o dos días- dolor lancinante en hombro derecho, seguido de debilidad para la abducción del mismo, cuadro que rápidamente se extiende a todo el miembro superior, afectando incluso a los movimientos finos de los dedos. A lo largo de las dos o tres semanas siguientes se instaura, de forma cada vez más ostensible, amiotrofia de las eminencias tenar e hipotenar, acentuándose la debilidad del miembro superior. Unas tres semanas más tarde, cuando empieza a remitir el dolor en hombro derecho, aparece idéntica sintomatología, aunque no tan intensa, en hombro izquierdo primero, y luego en todo el miembro superior izquierdo.

Se realizaron pruebas diagnósticas, incluyendo un estudio electrofisiológico, que reveló un patrón de denervación intenso afectando a C6, C7, C8 y D1; una radiografía de columna cervical, que mostró leves signos de artrosis, y una resonancia magnética en posición supina obteniendo una imagen axial potenciada en secuencia de inversión recuperación con saturación de la grasa (STIR, TR 6000, TE 34, TI $165 \mathrm{msec}$ ), realizando cortes de $10 \mathrm{~mm}$ de grosor, un espacio entre cortes de $0,5 \mathrm{~mm}$ y 2 adquisiciones (NEX 2), que mostró una señal hiperintensa que afectaba a la musculatura subescapular derecha, compatible con edema neurogénico; en el lado izquierdo se observó una lesión similar, aunque de menor intensidad (Fig. 1).

Con rehabilitación la sintomatología mejoró de forma lenta, desapareciendo primero la del lado izquierdo (al cabo de unos 2 meses) y luego la del derecho (a los 8-10 meses). La paciente completó además el tratamiento tuberculostático, habiéndose producido la fusión de D9 y D10 de forma total, encontrándose la paciente asintomática y sin datos de descompensación de su cirrosis.

\section{DISCUSIÓN}

La paciente presentaba un cuadro clasificable como síndrome de Parsonage-Turner bilateral, con afectación del plexo braquial, sobre todo en su porción inferior. Como antes señalamos, la afectación bilateral se ha descrito en un $25 \%$ de casos, habitualmente de forma sucesiva; a veces el proceso presenta recurrencias, aunque esto ocurre en menos del 5\% de casos (14). La patogenia es desconocida, aunque puede jugar un papel una respuesta inmune anormal. De hecho, el antecedente de una reacción inflamatoria intensa, una vacunación, de una infección vírica o bacteriana, o de

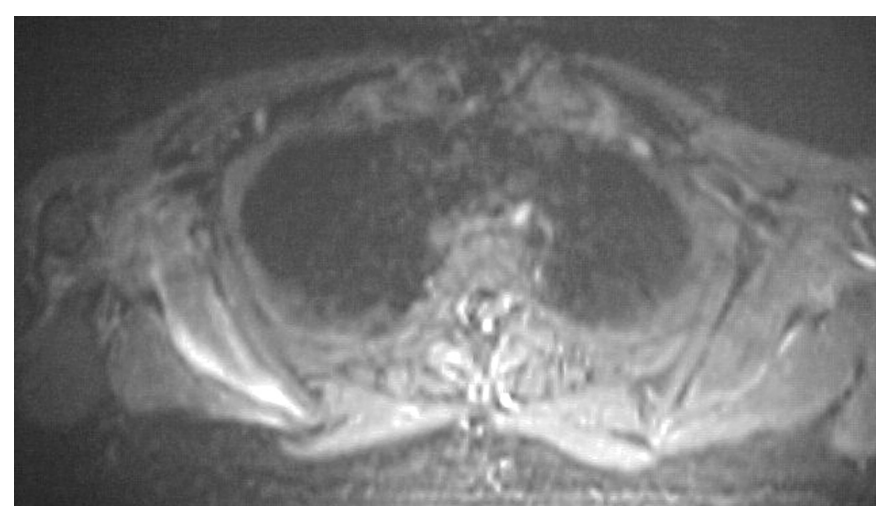

Fig. 1. Estudio axial de RNM, (secuencia STIR) mostrando una señal hiperintensa que afecta al músculo subescapular, compatible con edema neurogénico. Se aprecia una lesión similar, aunque menos marcada, en hombro izquierdo.

un proceso autoinmune o neoplásico se recoge en buen número de enfermos, como hemos señalado $(1,4,5,15)$. Tal es el caso de nuestra paciente, en la que una espóndilodiscitis tuberculosa dominaba el panorama clínico - aunque, que sepamos, no se ha descrito hasta ahora la tuberculosis en la lista de procesos relacionados con la neuralgia amiotrófica, aunque sí una lesión semejante en relación con un absceso frío retrofaríngeo (16). Cursa con dolor intenso localizado en hombro y región proximal del miembro superior, seguido de amiotrofia y pérdida de fuerza, en particular para la elevación y abducción del hombro, aunque puede predominar la afectación distal como ocurrió en el caso aquí presentado. Generalmente revierte en pocas semanas, aunque a veces pasan años hasta la recuperación total. El diagnóstico es clínico y electromiográfico, aunque recientemente se ha visto que la resonancia magnética proporciona una imagen altamente sugestiva (11-13). Los hallazgos más significativos consisten en la aparición de señales hiperintensas especialmente en músculos supra e infraespinoso o en otros de la cintura escapular, que se interpretan como derivadas del edema muscular secundario a la denervación. Los primeros estudios realizados con RNM en casos de Parsonage-Turner datan de 1998 (12). Probablemente este síndrome sea infradiagnosticado, ya que puede confundirse con la polineuropatía del paciente crítico si predomina la amiotrofia, y el paciente no percibe o expresa el dolor, o como secundario a problemas locales de la cintura escapular, si predomina el dolor, que, como ocurre habitualmente, suele ser de comienzo agudo y muy intenso. En este sentido, la RNM es de gran valor, ya que, además de ayudar a establecer el diagnóstico correcto, permite al tiempo excluir patología local, como tendinitis (un diagnóstico diferencial relevante en pacientes que, como la nuestra, estaba tratada con quinolonas), capsulitis, compresión o invasión tumoral del plexo braquial y otros problemas inflamatorios locales. 


\section{Bibliografía}

1. Vallejo Maroto I, Montes Latorre E, Giráldez Gallego A, Jiménez Macías FM, Herrera Justiniano JM. Neuralgia amiotrófica (síndrome de Parsonage Turner) de presentación bilateral en una paciente intervenida por abdomen agudo. Rev Clin Esp 2003; 203: 310.

2. Tsairis P, Dyck PJ, Mulder DW. Natural history of brachial plexus neuropathy: report on 99 patients. Arch Neurol, 1972; 27: 109-117.

3. Dananchet Y, Besson G. Syndrome de Parsonage et Turner et artérite de Horton. Rev Neurol (Paris) 2002; 158: 354-356.

4. Louis E, Touze E, Piketty ML, Salmon-Ceron D, Zuber M. Nevralgie amyotrophiante (syndrome de Parsonage-Turner) bilatérale révélatrice d'une séroconversion VIH. Rev Neurol (Paris) 2003; 159: 685-687.

5. Masson P, Rigot A, Cecile W. Brachial plexus neuropathy following pyogenic cervical adenophlegmon. Arch Pediatr 1994; 1: 735-737.

6. Lahrmann H, Grisold W, Authier FJ, Zifko UA. Neuralgic amyotrophy with phrenic nerve involvement. Muscle Nerve 1999; 22: 437-442.

7. Petit E, Deviere F, Tabaraud F, Troung T, Vallat JM, Couratier P. Une atteinte phrénique bilatérale revelatrice dún syndrome de Parsonage Turner. Rev Neurol (Paris) 2000; 156: 403-404.

8. Vanermen B, Aertgeerts M, Hoogmartens M, Fabry G. The syndrome of Parsonage and Turner. Discussion of clinical features with a review of 8 cases. Acta Orthop Belg 1991; 57: 414-419.

9. Misamore GW, Lehman DE. Parsonage-Turner syndrome (acute bra- chial neuritis). J Bone Joint Surg (Am.) 1996; 78: 1405-1408.

10. Stumpo M, Foschini MP, Poppi M, Cenacchi G, Martinelli P. Hypertrophic inflammatory neuropathy involving bilateral brachial plexus. Surg Neurol 1999; 52: 458-464.

11. Ryan M, Twair A, Nelson E, Brennan D, Eustace S. Whole body magnetic resonance imaging in the diagnosis of Parsonage Turner syndrome. Acta Radiol 2004; 45: 534-539.

12. Helms CA, Martínez S, Speer KP. Acute brachial neuritis (ParsonageTurner syndrome): MR imaging appearance-report of three cases. Radiology 1998; 207: 255-259.

13. Bredella MA, Tirman PF, Fritz RC, Wisher TK, Stork A, Genant HK. Denervation syndromes of the shoulder girdle: MR imaging with electrophysiologic correlation. Skeletal Radiol 1999; 28: 567572.

14. Michotte A, Dierckx R, Deleu D, Herregodts P, Schmedding E, Bruyland M, Ebinger G. Recurrent forms of sporadic brachial plexus neuropathy. A report of two cases. Clin Neurol Neurosurg 1988; 90: 71-74.

15. Simon JP, Fabry G.Parsonage-Turner syndrome after total-hip arthroplasty. J Arthroplasty, 2001; 16:518-520.

16. Valles H, García Guzmán A, Blanc JM, Fumanal L, de Francisco JE. Absceso frío tuberculoso retrofaríngeo. An Otorrinolaringol Ibero Am 1992; 19: 57-68. 